\author{
Langton S, Law AS, Burton AM \& Schweinberger SR (2008) \\ Attention capture by faces, Cognition, 107 (1), pp. 330-342.
}

This is the peer reviewed version of this article

NOTICE: this is the author's version of a work that was accepted for publication in Cognition. Changes resulting from the publishing process, such as peer review, editing, corrections, structural formatting, and other quality control mechanisms may not be reflected in this document. Changes may have been made to this work since it was submitted for publication. A definitive version was subsequently published in Cognition, [VOL 107, ISS 1 (2008)] DOI: http://dx.doi.org/10.1016/j.cognition.2007.07.012 


\title{
Attention capture by faces
}

\author{
Stephen R. H. Langton and Anna S. Law \\ Department of Psychology, University of Stirling, UK \\ A. Mike Burton \\ Department of Psychology, University of Glasgow, UK \\ Stefan R. Schweinberger \\ Institut für Psychologie, University of Jena, Germany
}

Address correspondence to: Dr. Stephen R. H. Langton, Department of Psychology, University of Stirling, Stirling, FK9 4LA, UK

Email: srhl1@stirling.ac.uk

Phone: (+44) (0)1786 467659 


\begin{abstract}
We report three experiments that investigate whether faces are capable of capturing attention when in competition with other non-face objects. In Experiment 1a participants took longer to decide that an array of objects contained a butterfly target when a face appeared as one of the distracting items than when the face did not appear in the array. This irrelevant face effect was eliminated when the items in the arrays were inverted in Experiment $1 \mathrm{~b}$ ruling out an explanation based on some low-level image-based properties of the faces. Experiment 2 replicated and extended the results of Experiment 1a. Irrelevant faces once again interfered with search for butterflies but, when the roles of faces and butterflies were reversed, irrelevant butterflies no longer interfered with search for faces. This suggests that the irrelevant face effect is unlikely to have been caused by the relative novelty of the faces or arises because butterflies and faces were the only animate items in the arrays. We conclude that these experiments offer evidence of a stimulus-driven capture of attention by faces.
\end{abstract}




\section{Introduction}

A typical natural scene contains many objects, few of which may be relevant to an organism's current behavioural goals. In order to know which objects are important each must be identified and its whereabouts in space established. Most contemporary theories of attention maintain that the visual system lacks the capacity to achieve full parallel identification of all items in the visual field. Instead, it is thought that a number of basic visual features can be identified preattentively and that this information is somehow integrated with top-down information to guide attention to locations of interesting objects in the visual field (e.g., Bundesen, 1990; Duncan \& Humphreys, 1989; Itti \& Koch, 2000; Treisman \& Sato, 1990; Wolfe, 1994). There is reasonable agreement that basic features such as colour, orientation, size and direction of movement are capable of preattentive analysis (see Wolfe, 1998, for a review). More complex properties such as 3-D structure (Enns \& Rensink, 1991), surfaces (He \& Nakayama, 1992), and stereoscopic depth (Nakayama \& Silverman, 1986) also seem capable of guiding attention and there is evidence that pre-attentive processes are sensitive to the occlusion of objects (Rensink \& Enns, 1998), suggesting that basic shape can be completed simultaneously at various locations across the visual field. However, there is rather less consensus concerning the ability of more complex shapes to guide attention in this bottom-up fashion.

Perhaps because of its biological and social significance, one particular shape - the human face - has been the focus of a good deal of work in this regard. Studies examining whether faces are able to capture attention have, generally speaking, operationalised capture as a performance benefit in a visual search task. In these studies, participants are generally asked to search a display for an upright (usually schematic) face among inverted face distracters. The time to find the target is measured as a function of the number of distracting 
elements in the display. The logic is that should a target item summon attention automatically, the number of distracting elements in a display will have a minimal effect on search time. However, the data do not generally support this conclusion (Kuehn \& Jolicoeur, 1994; Nothdurft, 1993). When researchers have used other objects as distracters as opposed to inverted faces, the results are more promising (Hershler \& Hochstein, 2005; Kuehn \& Jolicoeur, 1994, Experiment 3) though controversial (see the exchange between VanRullen, 2006 and Hershler \& Hochstein, 2006).

Notwithstanding the equivocal evidence for face capture in visual search, it is debateable whether the method itself is suitable for gauging attention capture. The problem is that the defining attribute of the target (i.e. it being a face) is the same as the reported attribute (i.e. yes, a face is present in the display). Thus it is probable that observers have somehow readied themselves to search for a particular type of target whereas true attention capture should occur in the absence of such readiness. As Yantis (1993) has pointed out, stimulus-driven attention capture should be defined as "attention capture by an attribute that is independent of either the defining or the reported attribute of the target" (p.677). With this in mind, the experiments reported here operationalise attention capture as a performance decrement produced by a taskirrelevant face. Following the additional singleton paradigm introduced by Theeuwes (e.g., Theeuwes, 1994) we asked participants to perform a visual search for a non-face target object (a butterfly) in arrays containing a total of six items. On half of the trials one of the distracting items was a face. If faces capture attention, their location will be visited on the majority of face-present trials and, on average, half of the remaining five locations in each array will also be searched before the target can be located (i.e., search through an average of 3.5 items). In face-absent trials, on average half of the six items will be searched in order to locate the target (i.e., search through an average of three items). We would therefore expect search time to be slower in arrays containing an irrelevant face compared with arrays where no face was 
present. The advantage of this method is that any such effect will be independent of both the defining and the reported attribute of the target (e.g., "butterfly" in both cases) and will thus fulfil Yantis's (1993) criterion for stimulus-driven attention capture.

\section{Experiments 1a and 1b}

In Experiment 1a participants were asked to search for butterfly amongst a circular display of six objects. On half of the trials the distracter items comprised instances of several different natural object categories. In the remaining trials a face appeared as one of the distracting objects. Experiment $1 \mathrm{~b}$ was identical to Experiment 1a in all respects except that the items in the arrays were inverted (i.e. rotated through 180 degrees). Inverting a face is thought to disrupt the configural processing of that face (e.g., Leder \& Bruce, 2000; Tanaka \& Farah, 1993) but will preserve low-level image-based properties such as edge density and local contrast which might make it salient in an array, or which it might share with the target item. Any effect of an irrelevant face which is caused by these low-level image properties should be preserved when the face is inverted.

\subsection{Method}

\subsubsection{Participants}

In each of Experiments 1a and $1 \mathrm{~b}$ participants were separate samples of 18 undergraduate students at the University of Glasgow, who received a small financial gratuity. All had normal or corrected-to-normal vision according to self report.

\subsubsection{Apparatus and materials}

Experiments ran on an Apple iMac personal computer with a 14 inch colour monitor using SuperLab software (Cedrus Corporation). The stimuli for Experiment 1a consisted of circular arrays of pictured greyscale objects on a white background, with a central fixation 
cross (see Figure 1). Objects were of different shapes but were sized to fit within a $3 \mathrm{~cm} \times 3$ $\mathrm{cm}$ square. The centre of each object was 4.3 degrees of visual angle from the central point of the display. A chin-rest was used to fix participants' viewing distance at $60 \mathrm{~cm}$ throughout the experiment. The target objects were six different pictures of butterflies. The distracter objects were varied exemplars from the categories of fruit, flowers, leaves, trees, houseplants, and faces. There were eight different distracter faces (four male and four female), all of which wore neutral expressions and were cropped to remove the external features of hair and ears.
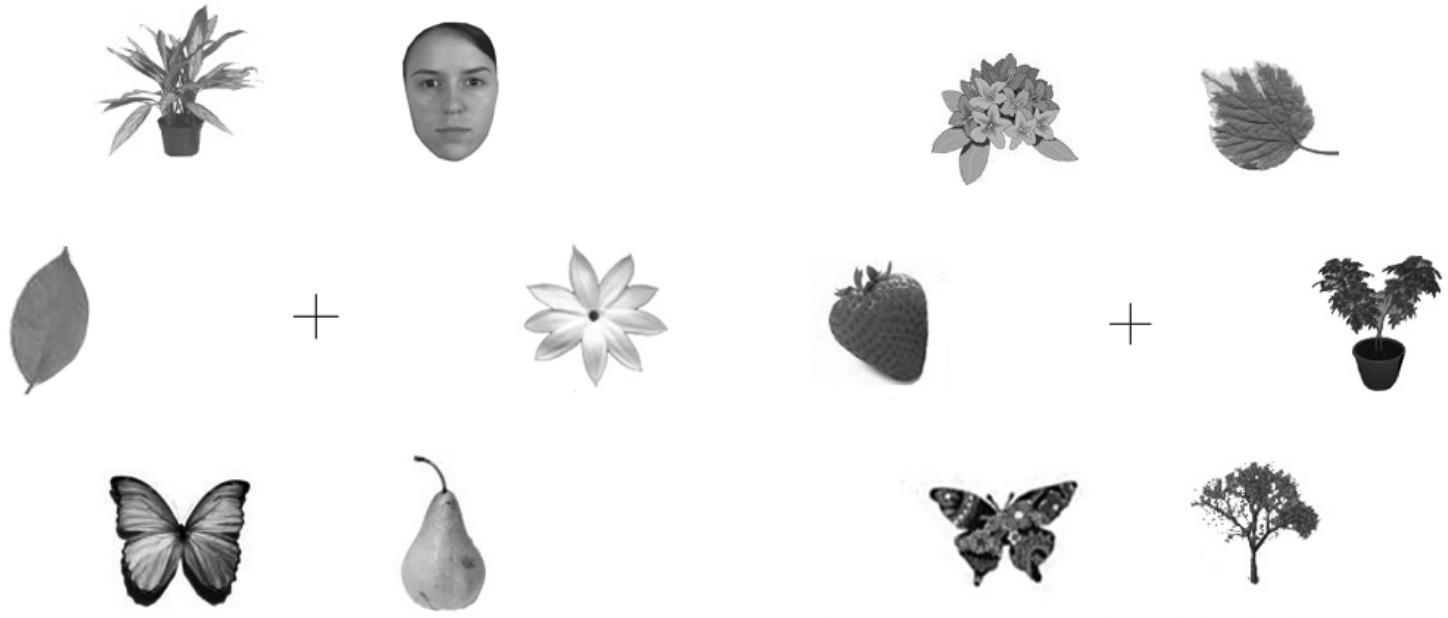

Figure 1. Examples of face-present (left panel) and face-absent (right panel) stimulus arrays used in the experiments. The butterfly items acted as targets in Experiments $1 \mathrm{a}$ and $1 \mathrm{~b}$ and the faces were targets in Experiment 2. The arrays were rotated through $180^{\circ}$ to produce the inverted stimuli in Experiment $1 b$.

Thirty arrays were created containing both a butterfly target and an irrelevant face. For each of the six possible target locations, an array was created with a face at each of the five remaining distracter locations, each with a unique butterfly-face pairing. The remaining distracter locations in each array were filled with objects selected at random from the five object categories. Thirty equivalent target present/face absent arrays were created by replacing the face in each array with an object which was randomly selected from one of the five categories with the constraint that an array could never contain two identical pictures. For 
each of the 60 target-present arrays, an equivalent target-absent array was created by replacing the butterfly with one of the other non-target objects, again with the constraint that an array could never contain two identical exemplars of the same object. Thus, in total there were 120 unique arrays.

\subsubsection{Design and Procedure}

The materials were tested in a within-subjects design with two factors: target (present vs. absent) and face (present vs. absent). Each trial began with a fixation cross which appeared in the centre of the screen for $1500 \mathrm{~ms}$ before the presentation of the stimulus array. Participants were asked to determine as quickly and accurately as possible whether or not a butterfly was present in each array and to make their responses by pressing either the " $\mathrm{z}$ " key or the " $\mathrm{m}$ " key on a standard keyboard which terminated the display. No mention was made of faces during instruction. Each of the 120 unique arrays was presented twice giving a total of 240 experimental trials. These were divided into two blocks of 120 trials, which were preceded by a block of 12 practice trials. Each test block contained an equal number of trials from each condition of the experiment, and the trials were presented in a different random order for each participant.

\subsection{Results and Discussion}

Median reaction times (RTs) were calculated for each of the four experimental conditions for every participant in each experiment. The inter-participant means of these median RTs along with the mean percentage of errors in each condition are summarised in Table 1. As predicted, reaction time to respond to the presence of a butterfly target in Experiment 1a was slower when an irrelevant face appeared in the display $(M=540 \mathrm{~ms})$ than when the face was absent $(M=520 \mathrm{~ms})$. An ANOVA conducted on the RT data for the upright arrays with Face (present vs. absent) and Target (present vs. absent) as within subjects factors yielded a 
significant main effect of Target, $F(1,17)=121.06, p<0.01$, and a significant interaction between Target and Face, $F(1,17)=11.44, p<0.01$. Simple main effects analyses confirmed that, for target-present trials, mean RT was significantly slower in arrays containing a face $(M$ $=540 \mathrm{~ms})$ than for arrays that $\operatorname{did} \operatorname{not}(M=520 \mathrm{~ms}), F(1,34)=9.62, p<0.01$, and that there was no effect of face for the target absent trials, $F(1,34)=1.54, p=0.22$. The time taken to detect the presence of a butterfly target was therefore increased by the inclusion of an irrelevant face in the display ${ }^{1}$. One interpretation of this finding is that the presence of a face captured attention so that, on average, more items needed to be searched in order to find the target in face-present compared to face-absent trials. The absence of a similar effect on targetabsent trials may be explained by participants engaging in a serial self-terminating search where all items must be searched - regardless of whether a face is present or not - before a negative response can be given.

Table 1

Mean reaction times (ms), percentage of errors and associated standard deviations (SD in brackets) in each of the conditions of Experiments $1 \mathrm{a}$ and $1 \mathrm{~b}$ where participants searched for butterfly targets.

\begin{tabular}{ccccccc}
\hline & & \multicolumn{2}{c}{ Target Present } & & \multicolumn{2}{c}{ Target Absent } \\
\cline { 3 - 4 } & & Face Present & Face Absent & & Face Present & Face Absent \\
\hline Experiment 1a & RT & $540(62)$ & $520(54)$ & & $649(83)$ & $657(88)$ \\
(Upright arrays) & $\%$ of errors & $5.83(3.87)$ & $4.63(3.41)$ & & $0.28(0.86)$ & $4.35(1.63)$ \\
Experiment 1b & RT & $618(139)$ & $619(136)$ & & $812(220)$ & $828(265)$ \\
(Inverted arrays) & $\%$ of errors & $5.09(3.81)$ & $4.82(2.48)$ & & $0.56(0.99)$ & $4.81(1.39)$ \\
\hline
\end{tabular}

Inspection of Table 1 also reveals that the target detection time in inverted arrays (Experiment $1 \mathrm{~b})$ was virtually unaffected by the presence $(M=619 \mathrm{~ms})$ or absence $(M=618$ ms) of the irrelevant face, an observation supported by the corresponding ANOVA which 
yielded only a significant main effect of target, $F(1,17)=42.51, p<0.01$ (all other $p$ 's $>0.1$ ).

Compared to face-absent arrays, the inclusion of an upright irrelevant face therefore slowed search for the target item whereas the inclusion of an inverted face produced no such effect ${ }^{2}$, ruling out explanations based on low-level image properties. The implication is that it is higher level representations of faces which produce the effect on visual search performance in upright arrays.

In addition to eliminating the influence of irrelevant faces on search for butterflies, inversion of the arrays also substantially slowed overall search time compared to search through upright arrays in Experiment $1 \mathrm{a}^{2}$. A good deal of research has suggested that basiclevel perceptual categorisation of both face and non-face items can be impaired when the stimuli are presented at various angles of rotation, including at $180^{\circ}$ (e.g., Rousselet, Macé \& Fabre-Thorpe 2003; Vannucci \& Viggiano, 2000), although the effects do seem to depend upon a variety of stimulus and task variables (Leek \& Johnston, 2006). Assuming the search task in our experiments involves basic level categorisation (i.e., face, butterfly, fruit etc) of each item after attentional selection then we might have predicted $a$ priori that visual search for butterflies in inverted arrays (Experiment 1b) would be slower on average than the equivalent search in upright arrays (Experiment 1a).

The main finding of this experiment is that the inclusion of upright but not inverted faces in the arrays slowed search for butterfly targets. This is consistent with the idea that faces capture attention ensuring that they are always searched when they appear in the displays. It is intuitive to imagine that, having initially sampling the face's location, search proceeds by attention selecting the adjacent items to the face and thereafter those that are progressively further from the face's location. However, our data offer no support for such a model; mean RTs for trials where targets were immediately adjacent to the face $(M=593 \mathrm{~ms})$, trials where 
targets were separated from the face by one item $(M=597 \mathrm{~ms})$ and by two items $(M=596)$ did not differ $(p=0.22)$. Instead, these data are more in line with models such as Guided Search (e.g., Wolfe, 1994) where attention is guided towards candidate target items in an order which is determined, not by location, but by a pre-attentive feature analysis. Items which share more features with the target of the search will receive priority over items which share fewer features. The distracting effect of irrelevant faces in the search task of Experiment 1a, however, suggests that faces (or features possessed by faces) will override this priority. Since the other items deemed to be potential targets on the basis of the pre-attentive analysis could occupy any of the locations in the arrays, adjacent locations to faces would be no more likely to be prioritised by attention than any of the other locations in the arrays.

Mean error rates across the two experiments were low $(3.77 \%$ and $3.82 \%$ respectively) and there was no systematic relationship between error scores and RT scores suggestive of a speed-accuracy trade-off. However, in both experiments error rates in the target absent conditions (i.e., false positives) were higher when the face was absent than when faces appeared in the arrays. Indeed, ANOVAs conducted on the error scores in both experiments revealed significant interactions between face and target [Experiment 1a: $F(1,17)=13.31, p$ $<0.01$. Experiment $1 \mathrm{~b}: F(1,17)=45.64, p<0.01]$. In each case simple main effects analyses confirmed that error rates for target absent trials were significantly higher when the face was absent compared to when it was present [Experiment 1a: $F(1,34)=23.13, p<0.01$ ); Experiment 1b: $F(1,34)=65.46, p<0.01]$ but that the face had no effect on error rates in target-present trials.

False positive errors in visual search tasks are generally fewer than miss errors and can be thought of as arising from trials where participants are uncertain or in which they make a guess, perhaps because of boredom, frustration, fatigue or anticipation (Chun \& Wolfe, 1996). 
In the absence of a face it seems that these guesses were more likely to be "yes" than "no" responses. One reason for this may be that some of the non-face distracter items shared more low-level features with the target butterflies than did the faces. On those trials where one of these items appeared instead of a face and where participants were guessing, the presence in the array of an item with a feature that also belongs to the target may have caused them to mistakenly guess that a target was present and respond accordingly.

Researchers studying attention make a distinction between attention capture in the sense of, on the one hand, attention being preferentially drawn to one particular item and, on the other, a delay in disengaging attention from a particular item once that item has been attended, whether or not it was preferentially attended in the first place (e.g., Fox, Russo, Bowles, \& Dutton, 2001). Indeed, recent research has suggested that faces may retain attention for longer than other objects in this latter sense (Bindemann, Burton, Hooge, Jenkins \& de Haan, 2005; Ro, Friggel \& Lavie, 2007). However, it seems unlikely that the effect noted in Experiment 1a could be attributed to this kind of disengagement difficulty as, presumably, this would have produced an effect of irrelevant faces in target-absent arrays and no such effect was evident. We therefore suggest that the irrelevant face effect arises because faces capture attention in the sense of drawing resources to their location.

However, there are at least two alternative explanations of the effect. First, it is possible that in performing the search for butterfly targets in Experiment 1a, participants adopted some attentional set which allowed other animate objects to be attended. Since faces are the only other of the items in the arrays which have this property, they may capture attention on some trials, not because they are faces, but because they happen to be animate objects ${ }^{3}$. Second, the irrelevant face effect observed in Experiment 1a may have arisen because of the relative novelty of the face distracters compared with the other distracter object categories in the 
arrays; faces appeared in 50 percent of the trials whereas exemplars from each of the remaining distracter object categories appeared in every trial. Experiment 2 was designed to investigate these possibilities.

\section{Experiment 2}

In Experiment 2, the arrays were identical to those used in Experiment 1a, but instead of being asked to search for butterflies, half of the participants were asked to search for faces and we investigated whether the presence of an irrelevant butterfly in the arrays would retard search times compared to search times in arrays where the butterfly distracter was absent. The remaining participants searched for butterflies in the presence or absence of an irrelevant face in what was effectively a replication of Experiment $1 \mathrm{a}$.

Once again, if faces capture attention we would expect to replicate the result of Experiment 1a: those participants searching for butterflies should be slowed by the presence of an irrelevant face in the display. Furthermore, we would expect those participants searching for faces to find them more rapidly than those searching for butterflies. However, if faces only capture attention because they share the property of animacy with the butterfly targets, we would also expect irrelevant butterflies to capture attention when faces are the target of the search while overall search time for faces would not necessarily be any faster than search for butterflies. Similarly, if the relative novelty of the face distracters compared to the other distracting items was responsible for the effect in Experiment 1a, then we would also expect the butterflies - which appeared equally as infrequently as did the faces in Experiment $1 \mathrm{a}$ - to capture attention and retard search time for faces.

\subsection{Method}

\subsubsection{Participants}

Forty-four undergraduates were recruited for Experiment 2; all had normal 
or corrected-to-normal vision and received course credit in exchange for their participation.

\subsubsection{Materials, Design and Procedure}

Participants in this experiment were assigned to either a face search task or a butterfly search task. The materials and procedure for the face search task were identical to those of Experiment 1a in all but the following respects. First, participants were asked to search the arrays for faces as opposed to butterflies. Second, in order to maintain the same ratio of exemplars from the target category to exemplars from the critical distracter category as used in Experiment 1a, six different faces were used as targets and eight different butterflies as the critical distracters. Participants assigned to the butterfly search task were presented with the same arrays and instructions as in Experiment 1a. Target Type (Face vs. Butterfly) was therefore a between subjects factor in this experiment and Distracter (present vs. absent) was treated as a within-subject factor.

\subsection{Results and Discussion}

The inter-participant means of participants' median RTs in each of the target present conditions of Experiment 2 are summarised in Table 2 along with the mean percentage of errors. Inspection of this table reveals that, as in Experiment 1a, search for butterflies was slowed by the inclusion of a face in the distracter set, although search for faces was unaffected by the presence or absence of a face distracter. Furthermore, search for faces was performed faster and with fewer errors than search for butterflies.

These observations were supported by the results of an ANOVA conducted on the target present RTs with Target Type (Face vs. Butterfly) as a between subjects variable and Distracter (present vs. absent) as a within-subjects variable. This analysis yielded a significant main effect of Target Type, $F(1,42)=7.59, p<0.01$ confirming that search for faces $(M=$ $516 \mathrm{~ms})$ was faster than search for butterflies $(M=573 \mathrm{~ms})$. However this main effect was 
qualified by a significant interaction between Target Type and Distracter, $F(1,42)=4.85, p<$ 0.05). Analysis of simple main effects confirmed that search was faster for faces than for butterflies regardless of whether a distracter was present, $F(1,84)=9.83, p<0.01$, or absent, $F(1,84)=4.70, p<0.05$. Most critically, however, it also confirmed that search for butterflies was slower when a face distracter was present $(M=582 \mathrm{~ms})$ than when it was absent $(M=$ $564 \mathrm{~ms}), F(1,42)=7.00, p<0.05$, but that search time for faces was equivalent whether a butterfly appeared in the display $(M=514 \mathrm{~ms})$ or not $(M=517 \mathrm{~ms}), F(1,42)=0.19, p=0.67$.

Table 2

Mean reaction times (ms), percentage of errors and associated standard deviations (SD in brackets) in each of the conditions of Experiment 2.

\begin{tabular}{|c|c|c|c|}
\hline & & Distracter Present & Distracter Absent \\
\hline & & \multicolumn{2}{|c|}{ Face Distracter } \\
\hline \multirow[t]{3}{*}{ Butterfly Target } & RT & $582(75)$ & $564(73)$ \\
\hline & $\%$ of errors & $3.48(3.70)$ & $3.41(3.19)$ \\
\hline & & \multicolumn{2}{|c|}{ Butterfly Distracter } \\
\hline \multirow[t]{2}{*}{ Face Target } & RT & $514(59)$ & $517(79)$ \\
\hline & $\%$ of errors & $1.59(1.82)$ & $1.67(1.36)$ \\
\hline
\end{tabular}

As can be seen in Table 2, the pattern of errors closely mirrored the RT data suggesting no trade-off between speed and accuracy. An equivalent ANOVA conducted on the error data yielded only a main effect of Target Type, $F(1,42)=6.22, p<0.05$ supporting the observation that more errors were made in searching for butterflies than in searching for faces.

Experiment 2 has therefore replicated the irrelevant face effect observed in Experiment 1a; however, the absence of any disrupting effect of an irrelevant butterfly on search for faces suggests that this face capture effect is unlikely to have been caused by the relative novelty of 
the faces or the shared animacy between faces and butterflies. Moreover, the finding that search for faces was significantly faster than search for butterflies is also in line with the idea that faces are prioritised by attention in visual search.

\section{General Discussion}

The aim of the experiments reported here was to determine whether faces are able to capture attention under conditions where the face was unrelated to the goal of the experimental task. In Experiment 1a participants' ability to search an array of objects for a target butterfly was slowed when an irrelevant face appeared in the array. This effect did not appear to be caused by low-level properties of the face images (Experiment 1b), nor by virtue of their novelty or by faces sharing the property of animacy with the butterfly targets (Experiment 2). Instead, and in line with other recent findings (Hershler and Hochstein, 2005; Theeuwes and Van der Stigchel, 2006), our results suggest that faces attract attention to their location and, furthermore, they seem capable of doing so in a bottom-up fashion since the faces in Experiment 1a were completely independent of both the defining and reported attributes of the target, fulfilling Yantis's (1993) criterion for stimulus-driven attention capture.

One way in which this face capture effect might be achieved is through top-down biasing of certain pre-attentively available features, or configuration of features, which may be possessed by faces but not by other non-face objects (e.g., Cave \& Batty, 2006). Perhaps extensive experience in searching for faces means that connections from the high-level face representations to the lower-level feature detectors are strengthened to such an extent that an item in an array which contains these features (i.e., a face) will have priority in a visual search whether it is the intended target item or not. Of course, this kind of mechanism would only lead to face capture if the relevant low-level features were not also possessed by other items 
in the display and must be able to account for the elimination of the effect when faces are inverted (Experiment 1b).

The above explanation rests on the expertise we all have with faces (see Bukach, Gauthier, \& Tarr, 2006); however, a second possibility, leaning more on a domain specificity account (see McKone, Kanwisher \& Duchaine, 2007), is that (upright) faces can actually be categorised preattentively, possibly because of neural circuitry dedicated to their analysis. In other words, faces may exist as structures in a preattentive view of the world which can be scrutinised by attentional processes which, in turn, operate to reveal more about these structures (e.g., their identities).

While not necessarily precluding the idea of preattentive face representations, there is an explanation of our irrelevant face effect which does not necessarily entail a capture of attention. Research has suggested that faces can be detected and categorised very efficiently by the visual system. For instance, Purcell and Stewart (1988) showed that under conditions of backward masking, the detection and discrimination of faces presented at fixation is achieved with shorter masking intervals than for upside down faces or jumbled faces made from the same features. A similar face detection effect was noted by Shelley-Tremblay and Mack (1999) who showed that smiley face icons were more resistant to metacontrast masking than scrambled or inverted faces. An implication of these findings is that we may become consciously aware of faces before other non-face items. Should this occur when a face appears in the search arrays in our experiments, the early conscious experience of the face may somehow retard responding to the target item, perhaps by delaying the initiation of search. On this view, the interfering effect of irrelevant faces is produced by the relative ease with which we become aware of faces rather than by their capturing of attentional resources during visual search. 
This is certainly an interesting idea, although its evaluation rather depends, in part, on where one stands with regard to the relationship between attention and consciousness. If one takes the view that attention is necessary for conscious experience (e.g., O'Regan \& Noe, 2001) then to say that one becomes aware of faces before other items is equivalent to saying that faces are attended before other items, which is precisely what we suggest. However, attention and consciousness are regarded by some as two rather different mechanisms of selection (e.g., Koch and Tsuchiya, 2006; Lamme, 2003). In this case, inputs to the system can be conscious or unconscious and either type of input can be processed with or without attention. The interference caused by irrelevant faces reported in our experiments might therefore be caused by preferential selection of faces by either consciousness or attention.

An experimental investigation of this hypothesis is beyond the scope of the present research; however, one argument against the consciousness view is that a face appearing in an array which did not otherwise contain a target might also be expected to reach awareness before the other items and delay the initiation of search. This version of the consciousness hypothesis would therefore predict that irrelevant faces ought to have an effect on both targetabsent as well as target-present displays, and yet the effect was only found in the latter. One might also question whether faces really do reach consciousness before other items. The experiments demonstrating the face detection effect show that face-like stimuli are detected more readily than control items matched with faces for their low-level image properties (inverted faces or jumbled faces); they do not show that faces are detected more efficiently than other non-face object categories (Purcell \& Stewart, 1988; Shelley-Tremblay and Mack. 1999). Whether or not faces reach awareness before other objects therefore remains an open question.

It is also worth noting that in Lamme's (2003) theory where attention and consciousness 
are regarded as distinct mechanisms, attention operates on inputs of which we may or may not be consciously aware and is required for the input to enter a form of visual awareness where its attributes may be reportable (e.g., access consciousness; see Block [2005]). In this sense, an early conscious representation of a face exists prior to the operation of visual selective attention. So, whether the irrelevant face effect is mediated by consciousness or - as we have argued here - by attention capture, its existence suggests the formation of what may be regarded as a preattentive representation. Wolfe (1998) has suggested that preattentive representations might encode basic categories such as "big" or "small", "steeply tilted" or "shallow", "green", "red" etc.; the research described here suggests that something like "face" or "non-face" might be added to the vocabulary describing this preattentive world. 


\section{References}

Bindemann, M., Burton, A.M., Hooge, I.T.C., Jenkins, R. \& de Haan, E.H.F. (2005). Faces retain attention. Psychonomic Bulletin \& Review, 12, 1048-1053.

Block, N. (2005). Two neural correlates of consciousness. Trends in Cognitive Sciences, 9, 46-52.

Bukach, C. M., Gauthier, I., \& Tarr, M. J. (2006). Beyond faces and modularity: the power of an expertise framework. Trends in Cognitive Sciences, 10, 159-166.

Bundesen, C. (1990). A theory of visual attention. Psychological Review, 97, 523-547.

Cave, K. R., \& Batty, M. J. (2006). From searching for features to searching for threat: drawing the boundary between preattentive and attentive vision. Visual Cognition, 14, 629-646.

Chun, M. M., \& Wolfe, J. M. (1996). Just say no: How are visual searches terminated when there is no target present? Cognitive Psychology, 30, 39-78.

Duncan, J., \& Humphreys, G. W. (1989). Visual search and stimulus similarity. Psychological Review, 96, 433-458.

Enns, J. T., \& Rensink, R. A. (1991). Preattentive recovery of three-dimensional orientation from line drawings. Psychological Review, 98, 335-351.

Fox, E., Russo, R., Bowles, R., \& Dutton, K. (2001). Do threatening stimuli draw or hold visual attention in subclinical anxiety? Journal of Experimental Psychology: General, $130,681-700$.

He. J. J., \& Nakayama, K. (1992). Surfaces vs features in visual search. Nature, 359, 231-233.

Hershler, O., \& Hochstein, S. (2004). At first sight: A high-level pop out effect for faces. 
Vision Research, 45, 1707-1724.

Hershler, O., \& Hochstein, S. (2006). With a careful look: still no low-level confound to face pop-out. Vision Research, 46, 3028-3035.

Itti, L., \& Koch, C. (2000). A saliency-based search mechanism for overt and covert shifts of visual attention. Vision Research, 40, 1489-1506.

Koch, C., \& Tsuchiya, N. (2006). Attention and consciousness: two distinct brain processes. Trends in Cognitive Sciences, 11, 16-22.

Kuehn, S. M., \& Jolicoeur, P. (1994). Impact of quality of image, orientation, and similarity of the stimuli on visual search for faces. Perception, 23, 95-122.

Lamme, V. A. F. (2003). Why visual attention and awareness are different. Trends in Cognitive Sciences, 7, 12-18.

Leder, H., \& Bruce, V. (2000). When inverted faces are recognised: The role of configural information in face recognition. Quarterly Journal of Experimental Psychology, 53A, 513536.

Leek, E. C., \& Johnston, S. J. (2006). A polarity effect in misoriented object recognition: The role of polar features in the computation of orientation-invariant shape representations. Visual Cognition, 13, 573-600.

McKone, E., Kanwisher, N., \& Duchaine, B. C. (2007). Can generic expertise explain special processing for faces? Trends in Cognitive Sciences, 11, 8-15.

Nakayama, K., \& Silverman, G. H. (1986). Serial and parallel processing of feature conjunctions. Nature, 320, 264-265.

Nothdurft, H. (1993). Faces and facial expressions do not pop out. Perception, 22, 1287-1298. 
O’Regan, J. K., \& Noë, A. (2001). A sensorimotor account of vision and visual consciousness. Behavioral and Brain Sciences, 24, 939-1031.

Purcell, D. G., \& Stewart, A. L. (1988). The face-detection effect: Configuration enhances detection. Perception \& Psychophysics, 43, 355-366.

Rensink, R. A., \& Enns, J. T. (1998). Early completion of occluded objects. Vision Research, $38,2489-2505$.

Ro, T., Friggel, A., \& Lavie, N. (2007). Attentional biases for faces and body parts. Visual Cognition, 15, 322-348.

Rousselet, G. A., Macé, M. J.-M., \& Fabre-Thorpe, M. (2003). Is it an animal? Is it a human face? Fast processing in upright and inverted natural scenes. Journal of Vision, 3, 440455.

Shelley-Tremblay, J., \& Mack, A. (1999). Metacontrast masking and attention. Psychological Science, 10, 508-515.

Tanaka, J. W., \& Farah, M. J. (1993). Parts and Wholes in face recognition. The Quarterly Journal of Experimental psychology, 46A, 225-245.

Theeuwes, J. (1994). Stimulus-driven capture and attentional set: selective search for color and visual abrupt onsets. Journal of Experimental Psychology: Human Perception \& Performance, 20, 799-806.

Theeuwes, J., \& Van der Stigchel, S. (2006). Faces capture attention: Evidence from inhibition of return. Visual Cognition, 13, 657-665.

Treisman, A., \& Sato, S. (1990). Conjunction search revisited. Journal of Experimental Psychology: Human Perception \& Performance, 16, 459-478.

VanRullen, R. (2006). On second glance: Still no pop-out effect for faces. Vision Research, 
46, 3017-3027.

Vannucci, M., \& Viggiano, M. P. (2000). Category effects on the processing of plane-rotated objects. Perception, 29, 287-302.

Wolfe, J. M. (1994). Guided Search 2.0: A revised model of visual search. Psychonomic Bulletin \& Review, 1, 202-238.

Wolfe, J. M. (1998). Visual search. In H. Pashler (Ed.). Attention (pp.13-74). Hove, UK: Psychology Press Ltd.

Yantis, S. (1993). Stimulus-driven attentional capture and attentional control settings. Journal of Experimental Psychology: Human Perception \& Performance, 19, 676-681. 


\section{Acknowledgements}

This research was supported by a Wellcome Trust grant (072308/B/03/Z) awarded to Burton, Schweinberger and Langton. Correspondence concerning this article should be addressed to Stephen R. H. Langton, Department of Psychology, University of Stirling, Stirling, FK9 4LA, Scotland. Electronic mail may be sent to srhl1@stirling.ac.uk.

We would like to thank Jeremy Wolfe and three anonymous reviewers for their helpful comments on an earlier version of this paper. 


\section{Footnotes}

1. We have repeated this procedure in an experiment where the target category was fruit and again search time was significantly slower in face-present $(M=632 \mathrm{~ms})$ versus face-absent arrays $(M=618 \mathrm{~ms} ; F(1,21)=6.80, p<0.05)$. In an additional experiment with a slightly modified procedure, participants were asked to make a 2-choice decision about an item of footwear (sports shoe or work shoe) which always appeared in the arrays containing a mix of natural and man-made objects. Once again, search time was slower in face-present $(M=807$ ms) versus face-absent arrays $(M=792 \mathrm{~ms})$ although the effect just failed to reach the conventional criterion for statistical significance $(F(1,22)=3.35, p=0.08)$.

2. An ANOVA conducted on the target present RT data from Experiments 1a and $1 \mathrm{~b}$ with orientation (upright vs. inverted) as a between-subjects factor and face (present vs. absent) as a within-subjects variable yielded the expected interaction between these factors, $F(1,34)=$ 10.73, $p<0.01$. Follow-up analysis of this interaction showed that the inclusion of a face slowed RTs in upright arrays $(520 \mathrm{~ms}$ vs. $540 \mathrm{~ms} ; F(1,34)=19.01, p<0.01)$, but had no effect when the arrays were inverted $(618 \mathrm{~ms}$ vs. $619 \mathrm{~ms} ; F(1,34)=0.048)$. The ANOVA also confirmed that search time for inverted arrays $(M=619 \mathrm{~ms})$ was significantly slower than for upright arrays $(M=530 \mathrm{~ms}), F(1,34)=6.36, p<0.05$.

3. A related explanation is that rather than capturing attention because they are grouped with target butterflies as animate objects, faces might represent a unique category in the arrays which otherwise contain items which could be grouped together as "natural objects". In other words it is uniqueness, rather than "faceness" per se, which captures attention. However, the finding, described above in footnote 1, that irrelevant faces also slowed RTs when participants searched arrays for shoe targets argues against this account. In this experiment the distracting objects were a mixture of natural objects (e.g., flowers, trees, fruit) and man-made 
items (e.g., musical instruments and houses) so that it is hard to see how a face could represent a unique category, other than by virtue of actually being a face. 